\title{
Is Drotrecogin alfa (activated) for adults with severe sepsis, cost-effective in routine clinical practice?
}

\author{
M Zia Sadique ${ }^{1 *}$, Richard Grieve 1 , David A Harrison², Brian H Cuthbertson ${ }^{3}$ and Kathryn M Rowan²
}

\begin{abstract}
Introduction: Previous cost-effectiveness analyses (CEA) reported that Drotrecogin alfa (DrotAA) is cost-effective based on a Phase III clinical trial (PROWESS). There is little evidence on whether DrotAA is cost-effective in routine clinical practice. We assessed whether DrotAA is cost-effective in routine practice for adult patients with severe sepsis and multiple organ systems failing.

Methods: This CEA used data from a prospective cohort study that compared DrotAA versus no DrotAA (control) for severe sepsis patients with multiple organ systems failing admitted to critical care units in England, Wales, and Northern Ireland. The cohort study used case-mix and mortality data from a national audit, linked with a separate audit of DrotAA infusions. Re-admissions to critical care and corresponding mortality were recorded for four years. Patients receiving DrotAA $(n=1,076)$ were matched to controls $(n=1,650)$ with a propensity score (Pscore), and Genetic Matching (GenMatch). The CEA projected long-term survival to report lifetime incremental costs per quality-adjusted life year (QALY) overall, and for subgroups with two or three to five organ systems failing at baseline.

Results: The incremental costs per QALY for DrotAA were $£ 30,000$ overall, and $£ 16,000$ for the subgroups with three to five organ systems failing. For patients with two organ systems failing, DrotAA resulted in an average loss of one QALY at an incremental cost of $£ 15,000$. When the subgroup with two organ systems was restricted to patients receiving DrotAA within 24 hours, DrotAA led to a gain of 1.2 QALYs at a cost per QALY of $£ 11,000$. The results were robust to other assumptions including the approach taken to projecting long-term outcomes.

Conclusions: DrotAA is cost-effective in routine practice for severe sepsis patients with three to five organ systems failing. For patients with two organ systems failing, this study could not provide unequivocal evidence on the costeffectiveness of DrotAA.
\end{abstract}

\section{Introduction}

Severe sepsis is the most common cause of death for patients admitted to critical care [1-3]. Recent international studies suggest that the annual incidence of severe sepsis is 50 to 100 cases per population of 100,000 [4]. Approximately $80 \%$ of critical care admissions with severe sepsis have multiple organ systems failing, and the associated hospital mortality is around $50 \%$. Severe sepsis is associated with substantial health-care costs; in

\footnotetext{
* Correspondence: zia.sadique@lshtm.ac.uk

'Department of Health Services Research and Policy, London School of Hygiene and Tropical Medicine, 15-17 Tavistock Place, London, WC1H 9SH, UK

Full list of author information is available at the end of the article
}

the US, the annual costs are approximately $\$ 17$ billion [1,5-9]. Severe sepsis survivors have a lower quality of life than the age- and sex-matched general population $[10,11]$.

There is ongoing debate about the effectiveness of severe sepsis therapies, including corticosteroids, intensive insulin therapy, and Drotrecogin alfa (activated) (DrotAA) (Xigris ${ }^{\circledR}$; Eli Lilly and Company, Indianapolis, IN, USA) (also known as a recombinant human activated protein $C$ ). In particular, although DrotAA has been evaluated in several large, multicenter randomized controlled trials (RCTs) [12,13], concerns remain about the therapy's effectiveness, both overall and for particular patient subgroups. In 2001, the PROWESS (Protein
C Biomed Central

() 2011 Sadique et al.; licensee BioMed Central Ltd. This is an open access article distributed under the terms of the Creative Commons Attribution License (http://creativecommons.org/licenses/by/2.0), which permits unrestricted use, distribution, and reproduction in any medium, provided the original work is properly cited. 
C Worldwide Evaluation in Severe Sepsis) trial reported that a 96-hour intravenous infusion $(24 \mu \mathrm{g} / \mathrm{kg}$ per hour) of DrotAA versus placebo reduced absolute mortality at 28 days by $6.1 \%$ [13]. However, subgroup analysis of the PROWESS trial suggested a benefit solely for 'high risk' patients, and the original US Food and Drug Administration (FDA) license was limited to this subgroup; for 'low risk' patients, concerns about side-effects and lack of benefit meant that a follow-up study was requested $[14,15]$. The European Medical Evaluation Agency (EMEA) license for DrotAA was granted under 'exceptional circumstances', indicating that the efficacy data were limited and annual reassessment was required. Both the FDA and the EMEA licensed DrotAA for use in patients with severe sepsis at high risk of death but differed in their definition of baseline risk of death. High risk of death was defined by the US label as an APACHE II (Acute Physiology and Chronic Health Evaluation II) score of 25 or more and by the European Union label as the presence of multiple organ failure. The subsequent ADDRESS (Administration of Drotrecogin alfa (activated) in Early Stage Severe Sepsis) trial included solely 'low risk' patients but was stopped early for futility [12]. A separate trial, RESOLVE (REsearching severe Sepsis and Organ dysfunction in children: a gLobal perspectiVE), was initiated to investigate the efficacy and safety of DrotAA in children [16] but did not report any beneficial effect of DrotAA and was also stopped early. A new RCT, 'PROWESS Shock' [17], will provide additional evidence on the efficacy of DrotAA for highrisk patients, specifically those with septic shock, but does not include a cost-effectiveness analysis (CEA).

For DrotAA, the relatively high drug costs - the acquisition cost of a 96-hour infusion is around $£ 5,900$ (2010 prices) - and associated critical care stay and the tradeoff between potential benefit and risk mean that rigorous CEA is particularly important. Previous studies reported that DrotAA was cost-effective for adults with severe sepsis [18-23], but because the studies were based on PROWESS, these findings may not apply to routine clinical practice. General concerns with the RCTs for DrotAA are that they were tightly regulated, included a narrow range of patients and centers, applied restrictive treatment protocols, focused on short-term endpoints (28-day mortality), and did not collect cost data. These concerns make the RCTs unsuitable for assessing the cost-effectiveness of DrotAA in routine clinical practice. Furthermore, previous studies did not assess the cost-effectiveness of DrotAA according to the baseline risk of death or the timing of DrotAA administration. Recent work has suggested that DrotAA may be more effective if given within 24 hours of admission $[24,25]$.
In the absence of appropriate RCTs, CEA may use observational data [26-28]. Recent observational studies have compared outcomes for severe sepsis patients receiving DrotAA with outcomes for matched controls [4,29-36]. However, none of these studies assessed costeffectiveness. We report cost-effectiveness of DrotAA by using data from a prospective cohort study that audited outcomes following DrotAA in routine clinical practice in England, Wales, and Northern Ireland [29].

This paper aims to assess the cost-effectiveness of DrotAA in routine clinical practice versus control for adult patients with severe sepsis and multiple organ systems failing. The results are presented both for the overall group (two to five organ systems failing within 24 hours of admission to the critical care unit) and for high- or low-risk subgroups, defined according to the number of organ systems failing (two or three to five).

\section{Materials and methods \\ Overview}

By extending a previous prospective cohort study [29], this CEA compared DrotAA versus no DrotAA (control) for adult patients who had severe sepsis or multiple organ systems failing and who were admitted to critical care units in England, Wales, or Northern Ireland. In brief, the previous study used audit data from routine clinical practice and applied inclusion and exclusion criteria based on PROWESS [13]. Data for each severe sepsis patient, either receiving DrotAA or not (controls), were linked to case mix, resource use, and cost data from a national audit. Patients receiving DrotAA were matched to controls. Readmissions to critical care and mortality were recorded for a follow-up period of 4 years. The study follows method guidelines and extrapolates from the observed data to report cost-effectiveness over the patients' lifetime [37]. Details of the data sources, estimation of costs and outcomes, and analytical methods are given below.

\section{Data source and DrotAA patients}

The effectiveness and costs for patients receiving DrotAA versus control were estimated with data from the Case Mix Programme (CMP) coordinated by the Intensive Care National Audit \& Research Centre (ICNARC). The CMP is a national comparative outcome audit and includes $91 \%$ of the adult general (mixed medical and surgical) critical care units (including intensive care, combined intensive care, and high-dependency care units) in England, Wales, and Northern Ireland. Prospective data on case mix, resource use, and outcomes were collected on consecutive admissions to each participating critical care unit (see Harrison and colleagues [38] for details). Case mix data were recorded within the first 24 hours following unit admission and include age, 
physiological measures, medical history, surgical status, and reason for admission. The physiology data are used to calculate important prognostic measures for case mix adjustment or subgroup analysis; these measures include acute physiology score (ICNARC model), baseline predicted probability of death (ICNARC model), and the number and type of organ systems failing within 24 hours following admission on the basis of PROWESS definitions. CMP data have been assessed to be of high quality and highly representative of all UK critical care units [39]. Support for the collection and use of patientidentifiable data without consent was obtained under section 60 of the UK Health and Social Care Act of 2001 (approval number PIAG 2-10[f]/2005).

In December 2002, ICNARC conducted a large, multicenter audit on the use of DrotAA and subsequent outcome. Overall, 112 units (57\% of those invited) actively participated; these units were representative of all those in the CMP. Each unit collected data on DrotAA use and adverse events for each admitted patient who received DrotAA at any time during their stay in the critical care unit. To provide CMP data on case mix, resource use, and outcomes, the DrotAA and CMP data were linked.

Patients who were admitted with severe sepsis and multi-organ systems failing or who developed severe sepsis and multi-organ systems failing during the first 24 hours in the critical care unit were identified by criteria derived from PROWESS [13]. Severe sepsis was defined as evidence of infection and three or more systemic inflammatory response syndrome criteria. Multiorgan systems failing was defined as two or more organ systems failing (cardiovascular, respiratory, renal, hematological, or metabolic) during the first 24 hours [29]. Each patient who met these criteria and received DrotAA at a participating unit during the recruitment period was included $(n=1,076)$.

\section{Controls}

Controls were CMP database patients who were defined as having severe sepsis and multiple organ systems failing during the first 24 hours following admission to critical care, according to the above criteria, but did not receive DrotAA. Of the four potential control pools considered in the previous study [29], the control pool judged $a$ priori to have the patients and units most similar to those receiving DrotAA was chosen. The control pool consisted of admissions contemporaneous to those who received DrotAA in those critical care units that went on to use DrotAA but who had not yet had their first use of DrotAA in that unit $(n=1,650)$.

\section{Costs}

All hospital costs for the DrotAA and controls were considered for the index hospital admission and for readmissions to the original critical care unit over the course of a 4-year period. Resource use data were recorded and combined with unit costs.

\section{Resource use measurement}

Resource use data were collected on the duration of DrotAA infusion, the length of stay (LOS) in critical care, and the total LOS in acute hospital. Information on the time at which DrotAA infusion was commenced was recorded. Complete data on the duration of DrotAA infusion were available for $90 \%$ of DrotAA cases; for the remainder, it was known that the infusion was interrupted; they were assumed to have the infusion for 48 , rather than 96, hours. Information on readmissions to the original critical care unit for a 4-year period, following the index admission, was available from the CMP for each DrotAA and control. Details on the number of readmissions and subsequent critical care and acute hospital LOSs were extracted. It was assumed that, after 4 years, there were no further morbidity costs attributable to the initial episode of severe sepsis [40].

\section{Unit costs}

Unit costs of DrotAA were taken from the National Institute for Health and Clinical Excellence (NICE) Health Technology Assessment (HTA) report [41]. For all admissions, each critical care bed-day was categorized according to the number of organ systems failing and costed with the corresponding cost per bed-day from the UK 'Payment by Results' database [42]. Costs per bed-day in hospital after discharge from critical care were taken from the literature [19]. All costs were adjusted to 2010-2011 price levels [43]. Resource use and unit costs were combined to report total costs per patient over the lifetime for DrotAA patients and for controls.

\section{Outcomes}

The main outcome measure was the quality-adjusted life year (QALY) over the patient's lifetime. This measure required using data on mortality from the original critical care unit admission and ensuing hospital episode, and from any subsequent readmissions to that critical care unit, to project life years for each patient. These estimated life years were combined with estimates of health-related quality of life (HRQOL) [44] to project lifetime QALYs for each patient.

\section{Mortality}

For the initial admission to critical care, information on vital status at acute hospital discharge and the date of death was taken from the CMP Database. For severe sepsis patients surviving the initial acute hospital episode, information on mortality following readmission to 
the same critical care unit, over 4 years, was used to reflect the excess risk of death in comparison with the general population $[40,45]$. For each patient whose death was recorded within those 4 years, the number of life years was then calculated directly (from the difference between date of death and date of initial admission). Patients who survived the initial acute hospital episode and all readmissions to the same critical care unit were assumed to have the same life expectancy, matched for age and gender, as the general population. There is evidence to suggest that severe sepsis survivors face a higher probability of death after the critical care episode in comparison with the general population (ageand gender-matched). However, there is no clear evidence of the duration of this excess mortality; the strongest evidence is in support of an excess mortality of up to 5 years, although some previous work has applied excess mortality for up to 25 years [46]. In our base case analysis, we follow previous studies $[40,45,47]$ and take a conservative approach and apply the excess mortality for up to 4 years (see subsequent sensitivity analysis).

\section{Health-related quality of life}

HRQOL for critical care survivors is lower than that for the general population after matching for age and gender $[45,47,48]$. We therefore down-weighted age- and gender-specific HRQOL weights from the general population [49]. We assumed, on the basis of the best available evidence from the literature [45], that HRQOL for critical care survivors was $80 \%$ that of the general population. We applied, on the basis of this long-term study, this adjustment factor for the 4 years following the initial admission, after which, HRQOL weights from the general population were applied (see subsequent sensitivity analysis, in which length of decrement of HRQOL is varied). Lifetime QALYs were reported by combining life years and HRQOL. Future QALYs and costs were discounted at the recommended rate of $3.5 \%$ [37].

\section{Analysis \\ Matching}

Matched cohort analyses were performed; initially, this was done according to a propensity score (Pscore). The Pscore model was similar to that developed previously [29] and estimated the probability of receiving DrotAA with a logistic regression model that included both patient and critical care unit level baseline factors. Unit factors included were hospital type and number of critical care beds. Patient factors included were as follows: age, ICNARC model physiology score (IMscore), gender, number of organ systems failing, specific organ failures (cardiovascular, respiratory, renal, hematological, and metabolic acidosis), source of admission to critical care (via the emergency department, theater or recovery, ward, clinic or home), diagnostic category (ICNARC Coding Method) [50], and serious condition in the medical history [29]. Age and IMscore were defined as nonlinear terms fitted as smoothed functions by using restricted cubic splines [51]; other continuous measures were assumed to have linear relationships with treatment assignment.

We then applied Genetic Matching (GenMatch), which extends Pscore matching by using an automated search algorithm to choose the best matches, which are those that maximize the balance in the distribution of baseline characteristics between the treatment and control groups [28,52-55]. The explicit aim of GenMatch is to maximize balance according to statistics such as $t$ tests or standardized differences in means. GenMatch matches on the Pscore but also matches on individual factors and can achieve better balance than matching on the Pscore alone [28,52-55]. A key advantage versus Pscore approaches is that GenMatch matches directly on any individual covariates judged to be potential confounders. Both methods matched one-to-one with replacement. So that average treatment effects could be estimated, each DrotAA case was matched to a control, and the remaining unmatched controls were each matched to a DrotAA case [56]. For both approaches, we reported balance on those variables previously judged to be important potential confounders [29]; these were age, gender, serious conditions in the medical history, acute physiology score (ICNARC model), predicted probability of acute hospital mortality (ICNARC model), proportion receiving mechanical ventilation, and number and types of organ systems failing during the first 24 hours in critical care. We reported covariate balance for the overall sample of DrotAA patients versus controls. Balance was measured with standardized mean difference; a difference of greater than $10 \%$ was taken to indicate meaningful imbalance $[57,58]$. Both matching methods were initially performed on the full sample of DrotAA patients and controls to report overall costeffectiveness.

We categorized patients a priori into two subgroups according to the number of organ systems failing during the first 24 hours in critical care: two organ systems failing (low-risk subgroup) and three to five organ systems failing (high-risk subgroup). These particular subgroup definitions were adopted previously $[29,41]$. We matched DrotAA patients and controls with both matching methods, separately for each subgroup, and reported the resultant balance statistics at the subgroup level.

\section{Statistical analysis}

The aim of the statistical analysis was to report the incremental effectiveness, costs, and cost-effectiveness of 
DrotAA versus control. We reported average treatment effect for the overall group of patients (two or more organ systems failing at baseline) and for the $a$ prioridefined subgroups of patients with two or with three to five organ systems failing. We reported the incremental effectiveness for DrotAA versus control as the odds ratio of acute hospital mortality at initial admission by applying logistic regression to the matched data. We compared mean costs and QALYs over the lifetime (DrotAA versus control). The CEA reported incremental cost-effectiveness ratios (ICERs) (costs per QALY) and incremental net benefits (INBs) [59] of DrotAA versus control. The INBs were calculated by valuing incremental QALYs according to the recommended level of willingness to pay for a QALY in the UK $(£ 20,000$ per QALY [37]) and subtracting from this the incremental costs.

Statistical uncertainty around the incremental results was considered by reporting $95 \%$ confidence intervals (CIs) by using the non-parametric bootstrap [60] on the matched data. One thousand bootstrap samples of the mean effectiveness and the mean costs were generated. The bootstrap recognized the correlation between costs and outcomes by bivariate re-sampling, and the re-sampling also stratified by treatment group. Incremental costs, incremental effects, and INB were calculated from the bootstrap sample. A cost-effectiveness acceptability curve [61] was constructed to report the probability that DrotAA was cost-effective at alternative levels of willingness to pay for a QALY gained.

\section{Sensitivity analysis}

The base case made the following assumptions that, while conservative, were judged to be potentially important: (a) GenMatch was the most appropriate matching method, (b) it was appropriate to include all DrotAA cases irrespective of the time infusion commenced, (c) DrotAA patients who did not have a complete duration of infusion and survived were assumed to have received $50 \%$ (48 hours) of the full infusion, (d) the excess mortality associated with severe sepsis was assumed for 4 years after critical care discharge, and (e) the decrement in quality of life for severe sepsis was applied for 4 years after hospital discharge. The sensitivity analyses tested whether the base case results were robust if the following alternative standpoints were taken:

Pscore matching To test whether the results were sensitive to the matching method, cost-effectiveness estimates were reported after Pscore matching rather than GenMatch.

Timing of DrotAA infusion This sensitivity analysis included only those DrotAA patients whose infusion was commenced within 24 hours of admission. This restricted sample was re-matched by using data from the original pool of controls. The main purpose of the sensitivity analysis was to assess whether the base case results were sensitive to potential for hidden bias in those treated with delay.

DrotAA drug cost It was assumed that patients who did not receive full infusion of DrotAA and were discharged alive from critical care received $75 \%$ (72 hours) of the full DrotAA infusion.

Duration of excess mortality The period of excess mortality was extended beyond the 4 years applied in the base case. Alternative data suggest that, for critical care survivors, excess mortality could continue for up to 25 years [46]. In this sensitivity analysis, the magnitude of excess death rates for an extended period of time (25 years) was taken from a previous study [46] and applied to patients who survived the initial acute hospital episode and all readmissions to the same critical care unit. These excess death rates relative to age- and gendermatched mortality in the general population were applied beyond 4 years for up to 25 years.

Health-related quality of life decrement The HRQOL decrement was assumed to be maintained up to 25 rather than 4 years [46].

Health-related quality of life of critical care survivors The HRQOL of critical care survivors was varied $70 \%$ to $90 \%$ to that of the general population rather than $80 \%$ assumed in the base case.

\section{Results}

\section{Covariate balance}

Before matching, DrotAA patients were, on average, younger (standardized difference of $29 \%$ ) and had a higher acute physiology score (standardized difference of $50 \%$ ) and a higher baseline probability of acute hospital death (standardized difference of 30\%) in comparison with controls (Table 1). After both Pscore matching and GenMatch, the baseline characteristics were similar between the treatment groups, but the standardized differences were generally lower following GenMatch (Table 1). For the subgroups of patients with two (Table 2a) and three to five (Table 2b) organ systems failing, patient characteristics were well balanced after GenMatch; additional covariates were also evenly distributed between the treatment groups (Additional data file 1). As GenMatch achieved better balance than Pscore matching, further results are reported for GenMatch only.

\section{Outcomes - mortality}

Table 3 reports odds ratios for the effect of DrotAA on acute hospital mortality within the initial admission. For the overall sample, the odds ratios of death for DrotAA versus control are less than 1 both before and after matching. For the subgroup with two organ systems failing, DrotAA was associated with increased hospital 
Table 1 Patient characteristics before and after propensity score matching and Genetic Matching

\begin{tabular}{|c|c|c|c|}
\hline & $\begin{array}{c}\text { Mean/percentage }^{a} \\
\text { DrotAA } \\
(n=1,076)\end{array}$ & $\begin{array}{c}\text { Mean/percentage }{ }^{a} \\
\text { Control } \\
(n=1,650)\end{array}$ & $\begin{array}{l}\text { Percent standardized } \\
\text { difference }\end{array}$ \\
\hline \multicolumn{4}{|l|}{ Age (year)s } \\
\hline Unmatched & 58.70 & 64.35 & 28.85 \\
\hline Pscore match & 61.48 & 61.90 & 2.55 \\
\hline GenMatch & 62.22 & 62.23 & 0.06 \\
\hline \multicolumn{4}{|c|}{ ICNARC model acute physiology score } \\
\hline Unmatched & 30.38 & 25.09 & 50.16 \\
\hline Pscore match & 27.24 & 27.18 & 0.63 \\
\hline GenMatch & 27.15 & 27.15 & 0.08 \\
\hline \multicolumn{4}{|c|}{ Serious conditions in medical history (percentage) } \\
\hline \multicolumn{4}{|c|}{ Cardiovascular } \\
\hline Unmatched & 0.47 & 1.82 & 11.63 \\
\hline Pscore match & 1.05 & 1.39 & 3.09 \\
\hline GenMatch & 1.28 & 1.28 & 0.00 \\
\hline \multicolumn{4}{|l|}{ Respiratory } \\
\hline Unmatched & 2.14 & 3.39 & 6.50 \\
\hline Pscore match & 2.71 & 3.02 & 1.85 \\
\hline GenMatch & 1.72 & 2.64 & 2.22 \\
\hline \multicolumn{4}{|l|}{ Renal } \\
\hline Unmatched & 1.12 & 2.36 & 8.31 \\
\hline Pscore match & 1.10 & 1.87 & 6.33 \\
\hline GenMatch & 1.14 & 1.94 & 2.32 \\
\hline \multicolumn{4}{|l|}{ Liver } \\
\hline Unmatched & 0.84 & 1.58 & 5.84 \\
\hline Pscore match & 5.53 & 1.52 & 21.88 \\
\hline GenMatch & 1.14 & 1.28 & 0.47 \\
\hline \multicolumn{4}{|l|}{ Immunosuppressed } \\
\hline Unmatched & 7.25 & 10.55 & 9.75 \\
\hline Pscore match & 11.35 & 9.80 & 5.06 \\
\hline GenMatch & 5.61 & 8.58 & 4.10 \\
\hline \multicolumn{4}{|c|}{ ICNARC model predicted probability of acute hospital mortality } \\
\hline Unmatched & 0.60 & 0.51 & 30.31 \\
\hline Pscore match & 0.55 & 0.55 & 0.21 \\
\hline GenMatch & 0.55 & 0.55 & 0.06 \\
\hline \multicolumn{4}{|c|}{$\begin{array}{l}\text { Number of organ systems failing during first } 24 \text { hours of stay in } \\
\text { critical care (percentage) }\end{array}$} \\
\hline \multicolumn{4}{|c|}{ Two } \\
\hline Unmatched & 18.40 & 38.18 & 38.20 \\
\hline Pscore match & 32.15 & 30.27 & 4.05 \\
\hline GenMatch & 29.35 & 29.79 & 0.34 \\
\hline \multicolumn{4}{|l|}{ Three } \\
\hline Unmatched & 40.06 & 38.91 & 1.91 \\
\hline Pscore match & 37.47 & 41.22 & 7.69 \\
\hline GenMatch & 38.11 & 41.16 & 2.20 \\
\hline \multicolumn{4}{|l|}{ Four } \\
\hline Unmatched & 33.55 & 18.12 & 28.31 \\
\hline Pscore match & 25.33 & 21.48 & 9.10 \\
\hline GenMatch & 26.85 & 22.71 & 3.40 \\
\hline \multicolumn{4}{|l|}{ Five } \\
\hline Unmatched & 7.99 & 4.79 & 10.33 \\
\hline Pscore match & 5.05 & 7.03 & 8.30 \\
\hline GenMatch & 5.69 & 6.35 & 0.98 \\
\hline
\end{tabular}




\begin{tabular}{|c|c|c|c|}
\hline \multicolumn{4}{|c|}{$\begin{array}{l}\text { Organ systems failing during first } 24 \text { hours of stay in critical care } \\
\text { (percentage) }\end{array}$} \\
\hline \multicolumn{4}{|c|}{ Cardiovascular/Respiratory } \\
\hline Unmatched & 14.03 & 25.27 & 24.24 \\
\hline Pscore match & 23.38 & 20.74 & 6.37 \\
\hline GenMatch & 23.51 & 20.73 & 2.38 \\
\hline \multicolumn{4}{|c|}{ Cardiovascular/Respiratory/Acidosis } \\
\hline Unmatched & 33.27 & 28.49 & 8.41 \\
\hline Pscore match & 28.49 & 32.32 & 8.33 \\
\hline GenMatch & 32.06 & 32.80 & 0.55 \\
\hline \multicolumn{4}{|c|}{ Cardiovascular/Respiratory/Renal/Acidosis } \\
\hline Unmatched & 25.37 & 12.30 & 26.50 \\
\hline Pscore match & 18.50 & 15.40 & 8.25 \\
\hline GenMatch & 19.15 & 16.25 & 2.69 \\
\hline \multicolumn{4}{|c|}{$\begin{array}{l}\text { Mechanical ventilation on admission or during first } 24 \text { hours of stay } \\
\text { in critical care (percentage) }\end{array}$} \\
\hline Unmatched & 92.47 & 75.33 & 42.51 \\
\hline Pscore match & 83.32 & 82.32 & 0.93 \\
\hline GenMatch & 82.25 & 82.21 & 0.03 \\
\hline
\end{tabular}

mortality, and the odds ratio after matching was 1.56 (95\% CI of 1.25 to 1.85 ). For the subgroup with three or more organ systems failing, DrotAA was associated with reduced hospital mortality (odds ratio following matching of $0.61,95 \% \mathrm{CI}$ of 0.53 to 0.79 ). The matching has balanced those potential confounders that are observed; for the subgroup with two organ systems failing, the result is that DrotAA is associated with an increase in hospital mortality in excess of $10 \%$.

The mean total costs of the initial hospital admission episode were about $£ 32,000$ and $£ 15,000$ for the DrotAA and control groups (Table 4). The additional costs for DrotAA comprised drugs costs but also additional days in critical care (mean of 15 versus 7.5 days for controls) (Additional data file 2). These additional days in critical care for DrotAA were observed for both survivors (mean of 17 versus 9 days) and decedents (mean of 11 versus 5 days). Approximately $10 \%$ of both DrotAA patients and controls were readmitted over the course of 4 years (Additional data file 3); hence, readmission costs were similar across the groups. Similarly, for both subgroups, DrotAA was associated with higher costs during the initial acute hospital stay (Table 4).

\section{Lifetime cost-effectiveness results}

The lifetime incremental cost-effectiveness results are reported in Table 5. For the overall sample, the incremental costs for DrotAA were $£ 18,000$ and the QALY gain was 0.60 , leading to an ICER of $£ 30,158$ per QALY.
The QALY gain of 0.6 valued at $£ 20,000$ led to an INB of $-£ 6,000(0.6 * 20,000-18,000)$. For patients with two organ systems failing, the higher mortality associated with DrotAA led to an average loss of one QALY, which, coupled with the positive incremental costs, led to a negative INB $(-£ 35,000)$. For the subgroup with three to five organ systems failing, the incremental QALY for DrotAA was relatively high (mean QALY gain of 1.3), the ICER was relatively low $(£ 15,561)$, and the corresponding INB was positive $(£ 6,000)$ (Table 5$)$. The statistical uncertainty around the CEA results is summarized in the cost-effectiveness acceptability curves in Figure 1. The probabilities that DrotAA is cost-effective at a threshold of $£ 20,000$ per QALY are $5 \%$ for the overall sample, $0 \%$ for the subgroup with two organ systems failing, and $99 \%$ for the subgroup with three or more organ systems failing.

\section{Sensitivity analysis}

The impact on the base case results of matching just with the Pscore rather than the individual covariates (GenMatch), of limiting DrotAA to within 24 hours, of increasing the drug cost, and of changing the duration of excess mortality and HRQOL decrement are shown in Table 6. When the sample was restricted to those who received DrotAA during the first 24 hours in critical care, the DrotAA cases were reduced by $36 \%$. For this subsample, acute hospital mortality following DrotAA was reduced, particularly for the subgroup with 
Table 2 Patient characteristics before and after Genetic Matching for each subgroup

\begin{tabular}{|c|c|c|c|}
\hline & $\begin{array}{c}\text { Mean/ } \\
\text { percentage }^{a} \\
\text { DrotAA }\end{array}$ & $\begin{array}{c}\text { Mean/ } \\
\text { percentage }^{\mathrm{a}} \\
\text { Control }^{-}\end{array}$ & $\begin{array}{l}\text { Percent standardized } \\
\text { difference }\end{array}$ \\
\hline a. Subgroup with two organ systems failing & $(n=198)$ & $(n=630)$ & \\
\hline \multicolumn{4}{|l|}{ Age (year)s } \\
\hline Unmatched & 57.58 & 63.04 & 26.49 \\
\hline GenMatch & 61.76 & 61.82 & 0.37 \\
\hline \multicolumn{4}{|l|}{ ICNARC model acute physiology score } \\
\hline Unmatched & 22.83 & 20.44 & 29.53 \\
\hline GenMatch & 20.86 & 20.88 & 0.35 \\
\hline \multicolumn{4}{|l|}{ Serious conditions in medical history, percentage } \\
\hline \multicolumn{4}{|l|}{ Cardiovascular } \\
\hline Unmatched & 1.01 & 1.75 & 5.40 \\
\hline GenMatch & 1.57 & 1.57 & 0.00 \\
\hline \multicolumn{4}{|l|}{ Respiratory } \\
\hline Unmatched & 4.55 & 4.13 & 1.67 \\
\hline GenMatch & 2.78 & 3.87 & 2.15 \\
\hline \multicolumn{4}{|l|}{ Renal } \\
\hline Unmatched & 2.02 & 3.81 & 9.17 \\
\hline GenMatch & 1.09 & 3.14 & 5.06 \\
\hline \multicolumn{4}{|l|}{ Liver } \\
\hline Unmatched & 1.52 & 1.27 & 1.69 \\
\hline GenMatch & 1.33 & 1.33 & 0.00 \\
\hline \multicolumn{4}{|l|}{ Immunosuppressed } \\
\hline Unmatched & 7.07 & 6.98 & 0.28 \\
\hline GenMatch & 4.59 & 6.16 & 2.46 \\
\hline \multicolumn{4}{|l|}{ ICNARC model predicted probability of acute hospital mortality } \\
\hline Unmatched & 0.42 & 0.39 & 10.76 \\
\hline GenMatch & 0.39 & 0.39 & 0.37 \\
\hline \multicolumn{4}{|c|}{ Organ systems failing in first 24 hours $^{\mathrm{b}}$ (percentage) - Cardiovascular/Respiratory } \\
\hline Unmatched & 76.26 & 66.19 & 18.61 \\
\hline GenMatch & 75.60 & 69.69 & 4.70 \\
\hline \multicolumn{4}{|l|}{ Mechanical ventilation (percentage) } \\
\hline Unmatched & 88.38 & 70.16 & 40.02 \\
\hline GenMatch & 75.24 & 75.12 & 0.10 \\
\hline b. Subgroup with three to five organ systems failing & $(n=878)$ & $(n=1,020)$ & \\
\hline \multicolumn{4}{|l|}{ Age (years) } \\
\hline Unmatched & 58.96 & 65.16 & 32.32 \\
\hline GenMatch & 62.46 & 62.47 & 0.00 \\
\hline \multicolumn{4}{|l|}{ ICNARC model acute physiology score } \\
\hline Unmatched & 32.08 & 27.96 & 40.83 \\
\hline GenMatch & 29.84 & 29.84 & 0.01 \\
\hline \multicolumn{4}{|l|}{ Serious conditions in medical history (percentage) } \\
\hline \multicolumn{4}{|l|}{ Cardiovascular } \\
\hline Unmatched & 0.34 & 1.86 & 13.58 \\
\hline GenMatch & 0.58 & 1.11 & 2.04 \\
\hline \multicolumn{4}{|l|}{ Respiratory } \\
\hline Unmatched & 1.60 & 2.94 & 7.78 \\
\hline GenMatch & 1.42 & 2.21 & 2.09 \\
\hline \multicolumn{4}{|l|}{ Renal } \\
\hline Unmatched & 0.91 & 1.47 & 4.39 \\
\hline GenMatch & 0.90 & 1.21 & 1.10 \\
\hline
\end{tabular}


Table 2 Patient characteristics before and after Genetic Matching for each subgroup (Continued)

\begin{tabular}{|c|c|c|c|}
\hline \multicolumn{4}{|l|}{ Liver } \\
\hline Unmatched & 0.68 & 1.77 & 8.70 \\
\hline GenMatch & 1.16 & 1.16 & 0.00 \\
\hline \multicolumn{4}{|c|}{ Immunosuppressed } \\
\hline Unmatched & 7.29 & 12.75 & 15.55 \\
\hline GenMatch & 6.11 & 9.80 & 4.83 \\
\hline \multicolumn{4}{|c|}{ ICNARC model predicted probability of acute hospital mortality } \\
\hline Unmatched & 0.64 & 0.58 & 20.12 \\
\hline GenMatch & 0.61 & 0.61 & 0.00 \\
\hline \multicolumn{4}{|c|}{$\begin{array}{l}\text { Number of organ systems failing during first } 24 \text { hours of stay in critical care } \\
\text { (percentage) }\end{array}$} \\
\hline \multicolumn{4}{|c|}{ Three } \\
\hline Unmatched & 49.09 & 62.94 & 22.88 \\
\hline GenMatch & 54.69 & 56.74 & 1.46 \\
\hline \multicolumn{4}{|l|}{ Four } \\
\hline Unmatched & 41.12 & 29.31 & 20.07 \\
\hline GenMatch & 36.57 & 34.14 & 1.79 \\
\hline \multicolumn{4}{|l|}{ Five } \\
\hline Unmatched & 9.80 & 7.75 & 5.82 \\
\hline GenMatch & 8.75 & 9.12 & 0.46 \\
\hline \multicolumn{4}{|c|}{ Organ systems failing during first 24 hours of stay in critical care ${ }^{b}$ (percentage) } \\
\hline \multicolumn{4}{|c|}{ Cardiovascular/Respiratory/Acidosis } \\
\hline Unmatched & 40.77 & 46.08 & 8.77 \\
\hline GenMatch & 44.10 & 43.99 & 0.08 \\
\hline \multicolumn{4}{|c|}{ Cardiovascular/Respiratory/Renal/Acidosis } \\
\hline Unmatched & 31.09 & 19.90 & 20.64 \\
\hline GenMatch & 25.76 & 24.97 & 0.64 \\
\hline \multicolumn{4}{|c|}{$\begin{array}{l}\text { Mechanical ventilation on admission or during first } 24 \text { hours of stay in critical } \\
\text { care (percentage) }\end{array}$} \\
\hline Unmatched & 93.39 & 78.53 & 38.90 \\
\hline GenMatch & 85.51 & 85.51 & 0.00 \\
\hline
\end{tabular}

a Mean reported for continuous variable and percentage for categorical variable.

${ }^{b}$ For the types of organ failure, results are presented for the most prevalent type for each number of organ failures; results for other types of organ failure are presented in Additional file 1. DrotAA, Drotrecogin alfa (activated); GenMatch, Genetic Matching; ICNARC, Intensive Care National Audit \& Research Centre.

two organ systems failing (Additional data file 4). Here, the odds ratio for DrotAA versus control was 0.64 (95\% CI of 0.50 to 0.78$)$, resulting in a QALY gain of 1.2 and an ICER of $£ 11,000$. As the accompanying cost-effectiveness acceptability curve shows, if the sample is limited to those commencing DrotAA within 24 hours, then the probability that the intervention is cost-effective exceeds 0.90 for both subgroups, irrespective of the willingness to pay for a QALY gained (Figure 2). The results were robust to the other main assumptions made in the base case (Table 6).

\section{Discussion}

This study reports the cost-effectiveness of DrotAA in routine clinical practice for adult severe sepsis patients admitted to critical care units in England, Wales, and Northern Ireland. The results suggest that DrotAA is cost-effective for patients at high risk of acute hospital death (three to five organ systems failing during the first 24 hours in critical care). For patients with two organ systems failing, the results are less clear; whereas the base case finding suggested that DrotAA was not costeffective, the sensitivity analysis suggested that the intervention was cost-effective if limited to those patients who received DrotAA within 24 hours.

This study extends previous CEAs of DrotAA by using data from a large sample of units delivering routine clinical care; previous CEAs used PROWESS data [18-23,62]. Our approach allows cost-effectiveness results to be presented for patients with characteristics relevant to a more general population with severe sepsis. We also report CEA results for patient subgroups defined according to the number of organ systems failing, reflecting different baseline risks of death. There is no consensus on how best to define risk for patients with severe sepsis; some recommendations, notably in 
Table 3 Acute hospital mortality and odds ratios for acute hospital mortality for all patients and for each subgroup before and after Genetic Matching

\begin{tabular}{|c|c|c|c|}
\hline & $\begin{array}{c}\text { DrotAA } \\
n / N \\
\text { (percentage) }\end{array}$ & $\begin{array}{c}\text { Control } \\
n / \mathrm{N} \\
\text { (percentage) }\end{array}$ & $\begin{array}{l}\text { Odds ratio }(95 \% \mathrm{Cl}) \text { for acute hospital mortality for DrotAA versus } \\
\text { control }\end{array}$ \\
\hline \multicolumn{4}{|c|}{$\begin{array}{l}\text { Overall: two to five organ systems } \\
\text { failing }\end{array}$} \\
\hline Unmatched & $\begin{array}{l}490 / 1,076 \\
(45.54)\end{array}$ & $\begin{array}{c}817 / 1,650 \\
(49.52)\end{array}$ & $0.85(0.72,0.98)$ \\
\hline GenMatch & $\begin{array}{l}508 / 1,076 \\
(47.25)\end{array}$ & $\begin{array}{l}875 / 1,650 \\
(53.04)\end{array}$ & $0.79(0.71,0.87)$ \\
\hline \multicolumn{4}{|c|}{ Two organ systems failing } \\
\hline Unmatched & $80 / 198(40.40)$ & $221 / 630(35.08)$ & $1.26(0.81,1.67)$ \\
\hline GenMatch & 93/198 (46.98) & $228 / 630(36.23)$ & $1.56(1.25,1.85)$ \\
\hline \multicolumn{4}{|c|}{ Three to five organ systems failing } \\
\hline Unmatched & $410 / 878(46.70)$ & $\begin{array}{l}596 / 1,020 \\
(58.43)\end{array}$ & $0.62(0.51,0.74)$ \\
\hline GenMatch & $423 / 878(48.16)$ & $\begin{array}{c}616 / 1,020 \\
(60.38)\end{array}$ & $0.61(0.53,0.69)$ \\
\hline
\end{tabular}

Cl, confidence interval; DrotAA, Drotrecogin alfa (activated); GenMatch, Genetic Matching.

the US, favor the use of APACHE II score [63], whereas others prefer the number of organ systems failing $[29,41]$. More generally, there is a clear consensus that, as in this study, subgroups should be defined a priori [41]. This study also extends previous CEAs of DrotAA by capturing important aspects of the longer-term impact of DrotAA in that readmissions and accompanying mortality are recorded for 4 years. This provides a more robust basis for extrapolating long-term outcomes than previous approaches $[18,19,21]$.

Table 4 Costs, in pounds sterling, of initial hospital episode and readmissions within 4 years

\begin{tabular}{|c|c|c|}
\hline & DrotAA, mean (SD) & Control, mean (SD) \\
\hline \multicolumn{3}{|c|}{ Overall: two to five organ systems failing } \\
\hline Drug cost & $5,022(1,688)$ & $0(0)$ \\
\hline ICU costs & $21,204(19,660)$ & $10,692(13,533)$ \\
\hline Hospital costs & $5,812(9,145)$ & $4,411(7,884)$ \\
\hline Total initial episode costs & $32,038(24,285)$ & $15,102(16,787)$ \\
\hline ICU readmission costs & $1,729(8,252)$ & $1,276(5,987)$ \\
\hline Hospital costs & $2,281(14,694)$ & $2,054(11,646)$ \\
\hline Total readmission costs & $4,010(21,380)$ & $3,330(16,057)$ \\
\hline \multicolumn{3}{|l|}{ Two organ systems failing } \\
\hline Drug cost & $5,328(1,338)$ & $0(0)$ \\
\hline ICU costs & $17,364(15,564)$ & $8,806(9,142)$ \\
\hline Hospital costs & $5,933(8,889)$ & $4,998(7,168)$ \\
\hline Total initial episode costs & $28,625(19,810)$ & $13,804(12,108)$ \\
\hline ICU readmission costs & $914(3,941)$ & $916(3,894)$ \\
\hline Hospital costs & $1,652(5,225)$ & $1,667(8,538)$ \\
\hline Total readmission costs & $2,567(8,202)$ & $2,684(11,308)$ \\
\hline \multicolumn{3}{|l|}{ Three to five organ systems failing } \\
\hline Drug cost & $4,916(1,773)$ & $0(0)$ \\
\hline ICU costs & $22,853(20,504)$ & $11,692(14,921)$ \\
\hline Hospital costs & $5,776(9,496)$ & $3,967(7,898)$ \\
\hline Total initial episode costs & $33,544(25,342)$ & $15,659(18,311)$ \\
\hline ICU readmission costs & $2,152(9,978)$ & $1,120(5,604)$ \\
\hline Hospital costs & $2,823(20,799)$ & $1,794(11,361)$ \\
\hline Total readmission costs & $4,976(28,531)$ & $2,914(15,488)$ \\
\hline
\end{tabular}

Overall (two to five organ systems failing): total number $=2,726$, Drotrecogin alfa (activated) (DrotAA) $=1,076$, control $=1,650$; two organ systems failing: total number $=828$, Drot $A A=198$, control $=630$; three to five organ systems failing: total number $=1,898$, Drot $A A=878$, control $=1,020$. ICU, intensive care unit. 
Table 5 Lifetime costs in pounds sterling, quality-adjusted life years, and incremental net benefits in pounds sterling after Genetic Matching

\begin{tabular}{|c|c|c|c|}
\hline & DrotAA, mean (SD) & Control, mean (SD) & Incremental, mean $(95 \% \mathrm{Cl})$ \\
\hline \multicolumn{4}{|c|}{ Overall: two to five organ systems failing } \\
\hline Lifetime costs & $36,048(35,522)$ & $18,432(26,708)$ & $17,616(15,959$ to 19,273$)$ \\
\hline Lifetime QALYs & $5.70(6.57)$ & $5.11(6.61)$ & $0.58(0.24$ to 0.93$)$ \\
\hline$I^{N} B^{a}$ & $77,896(131,138)$ & $83,830(129,324)$ & $-5,934(-12,735$ to 868$)$ \\
\hline \multicolumn{4}{|c|}{ Two organ systems failing } \\
\hline Lifetime costs & $31,191(21,959)$ & $16,488(18,399)$ & $14,703(12,763$ to 16,644$)$ \\
\hline Lifetime QALYs & $5.87(6.54)$ & $6.83(6.75)$ & $-0.97(-1.62$ to -0.32$)$ \\
\hline $\mathrm{INB}^{\mathrm{a}}$ & $86,117(130,821)$ & $120,148(134,047)$ & $-34,031(-47,028$ to $-21,034)$ \\
\hline \multicolumn{4}{|c|}{ Three to five organ systems failing } \\
\hline Lifetime costs & $38,520(43,205)$ & $18,572(27,266)$ & $19,948(17,610$ to 22,286$)$ \\
\hline Lifetime QALYs & $5.63(6.59)$ & $4.35(6.33)$ & $1.28(0.86$ to 1.70$)$ \\
\hline $\mathrm{INB}^{\mathrm{a}}$ & $74,038(132,559)$ & $68,348(122,496)$ & $5,690(-2,543$ to 13,924$)$ \\
\hline
\end{tabular}

Overall (two to five organ systems failing): total number $=2,726$, Drotrecogin alfa (activated) (DrotAA) = 1,076, control = 1,650; two organ systems failing: total number $=828$, DrotAA $=198$, control $=630$; three to five organ systems failing: total number $=1,898$, DrotAA $=878$, control $=1,020$. ${ }^{a}$ Incremental net benefits (INBs) are calculated by following method guidance [36] and multiplying the mean quality-adjusted life year (QALY) gain (or loss) by $£ 20,000$ and subtracting from this the incremental cost. $\mathrm{Cl}$, confidence interval; SD, standard deviation.

A further strength of this study is that it uses appropriate methods to address selection bias by applying both Pscore matching and GenMatch, which are recommended approaches for addressing baseline differences between treatment groups in potential confounding factors. Both Pscore and GenMatch achieved good balance on key prognostic factors, and GenMatch achieved excellent balance both overall and for each subgroup. Hence, the possibility of bias arising from differences in observed factors was minimized.

Short-term mortality estimates of this study are broadly consistent with those from PROWESS. We

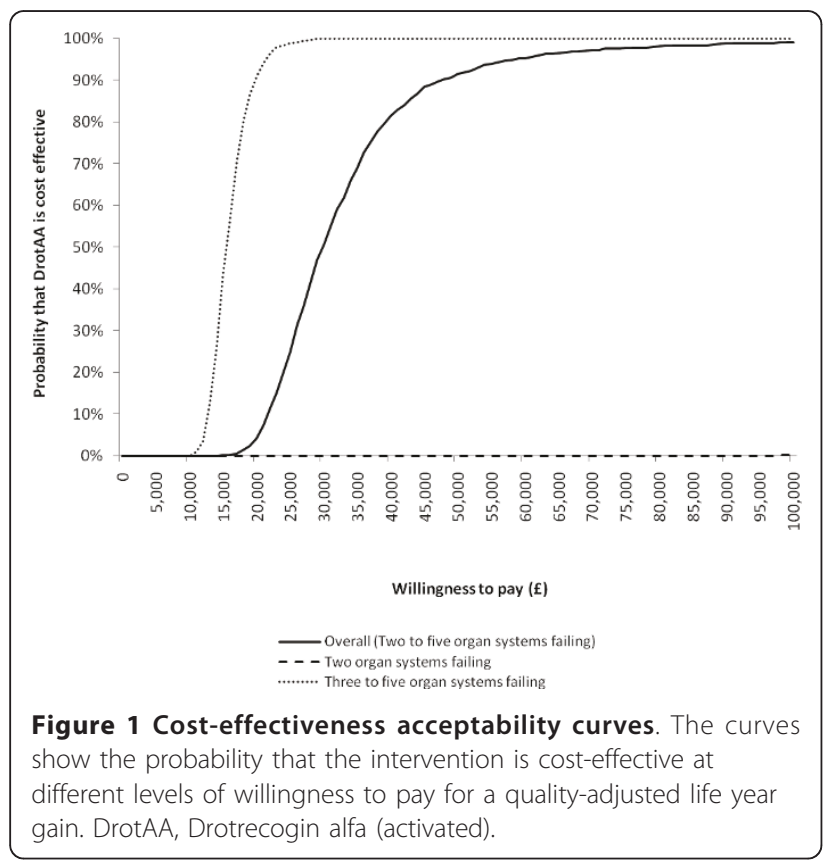

found that DrotAA reduced absolute acute hospital mortality by $5.8 \%$ in comparison with a $6.1 \%$ reduction in 28-day mortality in the PROWESS study. Our odds ratios $(0.79,95 \%$ CI 0.71 to 0.87$)$ are slightly less favorable for DrotAA than in PROWESS $(0.8,95 \%$ CI 0.69 to 0.94). The somewhat smaller effect of DrotAA on mortality leads to lower lifetime QALY gains compared with those of previous studies based on PROWESS $[18,19,21,22,62]$. Our lifetime incremental costs of DrotAA $(£ 18,000)$ exceeded previous estimates (less than $£ 6,000)[19,21,62]$, which reflected the relatively high initial LOS for DrotAA patients managed in routine clinical practice (mean of 15 days for DrotAA versus 7.5 days for the control group). Consequently, our estimates of the long-term cost-effectiveness of DrotAA are less favorable than those previously reported $[18,22]$. Dhainaut and colleagues [64], applying Pscore matching, also observed less favorable cost-effectiveness ratios; the authors reported a cost per QALY of $€ 34,000$ in routine clinical practice in France.

When methodological guidelines are followed and cost-effectiveness results are reported over the lifetime, assumptions inevitably have to be made. Our sensitivity analysis suggested that the findings, with one exception, were generally insensitive to the assumptions made. The base case results reported that DrotAA was not costeffective for the subgroup with two organ systems failing. If the sample is limited to those cases receiving DrotAA administered early (within 24 hours of admission), then the intervention, on average, reduces mortality for the subgroup with two organ systems failing and becomes cost-effective. Several other studies have indicated the benefit of early treatment. The ENHANCE (Extended Evaluation of Recombinant Human Activated 
Table 6 Sensitivity analysis on incremental net benefits in pounds sterling

\begin{tabular}{|c|c|c|c|}
\hline & $\begin{array}{l}\text { Overall: two to five organ } \\
\text { systems failing }\end{array}$ & Two organ systems failing & $\begin{array}{l}\text { Three to five organ } \\
\text { systems failing }\end{array}$ \\
\hline Base case & $-5,934(-12,735$ to 868$)$ & $-34,031(-47,028$ to $-21,034)$ & $5,690(-2,543$ to 13,924$)$ \\
\hline Pscore matching & $-7,641(-13,213,-2,069)$ & $-32,846(-44,704,-20,987)$ & $391(-6,350,7,133)$ \\
\hline DrotAA given within 24 hours $^{\mathrm{a}}$ & $8,078(733$ to 15,423$)$ & $11,131(-2,173$ to 24,435$)$ & $12,387(3,491$ to 21,283$)$ \\
\hline Cost of drug (alternative assumption) & $-4,687(-11,544$ to 2,171$)$ & $-33,232(-46,309$ to $-20,154)$ & $7,339(-976$ to 15,653$)$ \\
\hline Excess mortality up to 25 years & $-6,240(-12,897$ to 418$)$ & $-33,580(-46,300$ to $-20,860)$ & $5,046(-3,012$ to 13,104$)$ \\
\hline Excess reduction in $\mathrm{HRQOL}$ up to 25 years & $-7,240(-13,328$ to $-1,152)$ & $-31,895(-43,509$ to $-20,280)$ & $2,863(-4,512$ to 10,237$)$ \\
\hline $\begin{array}{l}\text { HRQOL of critical care survivors is } 70 \% \text { that of } \\
\text { general population }\end{array}$ & $-6,029(-12,679$ to 621$)$ & $-32,176(-44,912$ to $-19,440)$ & $4,839(-3,202$ to 12,880$)$ \\
\hline $\begin{array}{l}\text { HRQOL of critical care survivors is } 90 \% \text { that of } \\
\text { general population }\end{array}$ & $-5,347(-12,254$ to 1,560$)$ & $-33,134(-46,353$ to $-19,915)$ & $6,239(-2,117$ to 14,596$)$ \\
\hline
\end{tabular}

Values are presented as mean ( $95 \%$ confidence interval). Incremental net benefits are calculated by multiplying the mean quality-adjusted life year gain (or loss)

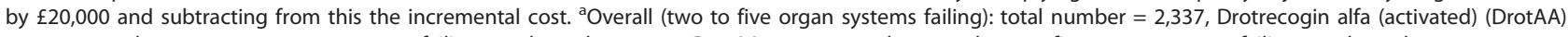
$=687$, control = 1,650; two organ systems failing: total number $=727$, DrotAA $=97$, control $=630$; three to five organ systems failing: total number $=1,610$, $\operatorname{Drot} A \mathrm{~A}=590$, control $=1,020$. HRQOL, health-related quality of life; Pscore, propensity score.

Protein C) trial showed that treatment within 24 hours of organ failure with DrotAA was associated with $23 \%$ lower odds of death at 28 days in comparison with treatment more than 24 hours after sepsis-induced organ failure [25]. Others also have suggested that DrotAA is more effective when administered early [24,29].

This study has several limitations. Firstly, we assumed that acute hospital survivors who were not readmitted to critical care faced a death rate equal to that of the general population (age- and gender-matched), and this may have underestimated deaths in this specific group of patients. Our estimated death rates were similar to those used previously; for example, our overall projected mortality at 5-

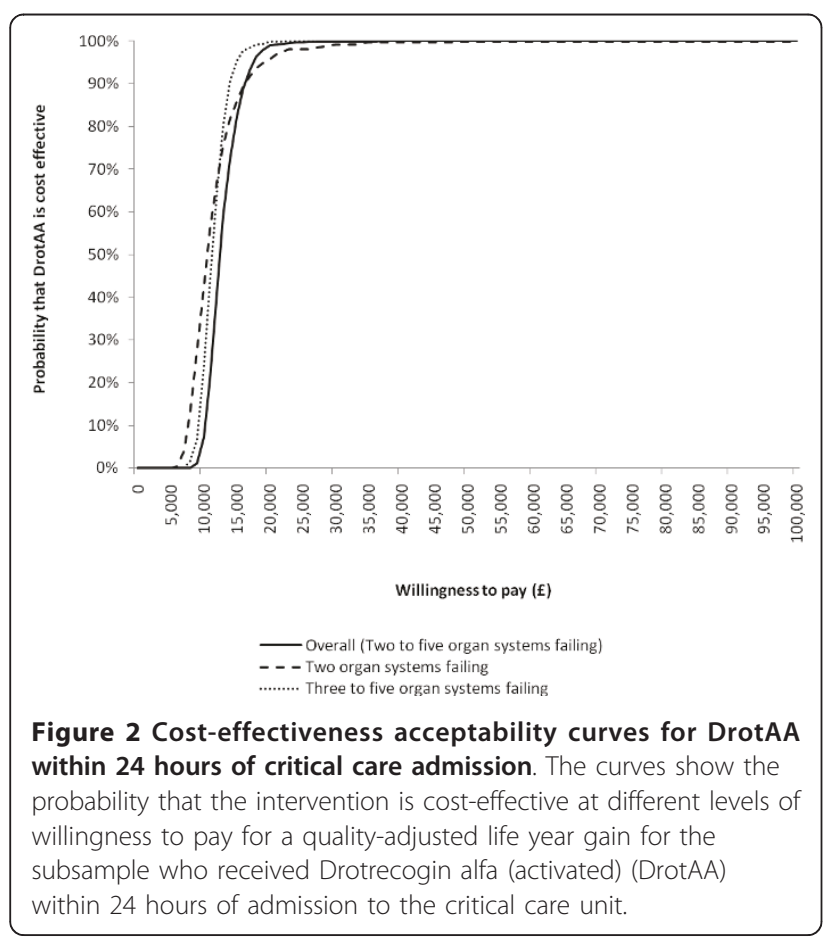

year follow-up was $49 \%$ versus $47 \%$ in Wright and colleagues [40]. Furthermore, the sensitivity analysis finds that the CEA results are insensitive to this assumption. Secondly, like other investigators attempting to report lifetime cost-effectiveness, we had to make plausible assumptions about the long-term HRQOL; we tested these assumptions in the sensitivity analysis and found that they had little impact on the results. Thirdly, unbiased treatment effects from matching are based on the assumption of no hidden bias; this assumption might be less tenable for the subgroup with two organ systems failing, in which $36 \%$ of the DrotAA cases are treated after a delay of more than 24 hours. There is some limited evidence from the FDA post hoc analysis [15] and ADDRESS trial [12] to suggest that DrotAA may increase mortality in subgroups with low baseline risk. However, our sensitivity analysis suggested that, if the sample was limited to those treated within 24 hours, DrotAA was more effective and was cost-effective for the subgroup with two organ systems failing. Here, the case severity at the time DrotAA infusion commenced might have been worse than that measured at baseline and used in the matching. This suggests that either the base case findings for the subgroup with two organ systems failing is prone to hidden bias or DrotAA is more effective and costeffective for this subgroup if administered early. Further research is required to focus specifically on the effectiveness and cost-effectiveness of DrotAA administration for patients at low levels of baseline severity and according to the timing of therapy initiation.

\section{Conclusions}

This CEA, based on a prospective cohort study, suggests that, in routine clinical practice, DrotAA is cost-effective for severe sepsis patients with three or more organ systems failing within 24 hours of admission to a critical care unit. For patients with two organ systems failing, 
this study could not provide unequivocal evidence on the cost-effectiveness of DrotAA.

\section{Key messages}

- Drotrecogin alfa (activated), or DrotAA, is costeffective in routine practice for severe sepsis patients with three to five organ systems failing during the first 24 hours in critical care.

- For patients with two organ systems failing, it is unclear whether DrotAA is cost-effective.

- Further research is required on the effectiveness and cost-effectiveness of DrotAA administration for patients at low levels of baseline severity and according to the timing of therapy initiation.

\section{Additional material}

\begin{tabular}{|c|}
\hline $\begin{array}{l}\text { Additional file 1: Additional patients' characteristics before and } \\
\text { after Pscore matching and GenMatch. Balance statistics of additional } \\
\text { covariates are shown. }\end{array}$ \\
\hline $\begin{array}{l}\text { Additional file 2: Length of stay (days) in critical care at index } \\
\text { admission - mean (sd). Shown is the length of stay in critical care in } \\
\text { DrotAA and control before and after GenMatch. }\end{array}$ \\
\hline $\begin{array}{l}\text { Additional file 3: Readmission to original critical care unit within } 4 \\
\text { years }(\mathrm{n} / \mathrm{N}(\%)) \text { and mortality at readmission }(\mathrm{n} / \mathrm{N}(\%)) \text {. Readmissions } \\
\text { and observed deaths in four years in original critical care unit are shown. }\end{array}$ \\
\hline $\begin{array}{l}\text { Additional file 4: Odds ratio, lifetime costs }(£) \text {, QALYs, incremental } \\
\text { net benefits (INB) }(£) \text { mean }(95 \% \mathrm{CI}) \text { for the subsample who } \\
\text { received DrotAA within } 24 \text { hours of admission to the critical care } \\
\text { unit. Odds ratio, lifetime costs, QALYs, and incremental net benefits are } \\
\text { shown where DrotAA is given within } 24 \text { hours of admission. }\end{array}$ \\
\hline
\end{tabular}

\section{Abbreviations}

ADDRESS: Administration of Drotrecogin alfa (activated) in Early Stage Severe Sepsis; APACHE II: Acute Physiology and Chronic Health Evaluation II; CEA: cost-effectiveness analysis; Cl: confidence interval; CMP: Case Mix Programme; DrotAA: Drotrecogin alfa (activated); EMEA: European Medical Evaluation Agency; FDA: US Food and Drug Administration; GenMatch: Genetic Matching; HRQOL: health-related quality of life; ICER: incremental cost-effectiveness ratio; ICNARC: Intensive Care National Audit \& Research Centre; IMscore: Intensive Care National Audit \& Research Centre model physiology score; INB: incremental net benefit; LOS: length of stay; PROWESS: Protein C Worldwide Evaluation in Severe Sepsis; Pscore: propensity score; QALY: quality-adjusted life year; RCT: randomized controlled trial.

\section{Acknowledgements}

We gratefully acknowledge Noemi Kreif and Rosalba Radice (both of the London School of Hygiene and Tropical Medicine) and Roland Ramsahai (University of Cambridge) for their useful advice on data analysis and comments on an earlier draft of the manuscript.

\section{Author details}

${ }^{1}$ Department of Health Services Research and Policy, London School of Hygiene and Tropical Medicine, 15-17 Tavistock Place, London, WC1H 9SH, UK. ${ }^{2}$ Intensive Care National Audit \& Research Centre, Tavistock House, Tavistock Square, London, WC1H 9HR, UK. ${ }^{3}$ Department of Critical Care Medicine, Sunnybrook Health Sciences Centre, Bayview Avenue, Toronto, ON, M4N 3M5, Canada.

\section{Authors' contributions}

ZS and RG participated fully in the design of this study, the analysis of the data, and the writing of this paper. DAH and KMR participated fully in the design of this study, the collection of data, the analysis of the data, and the writing of this paper. $\mathrm{BHC}$ participated fully in the analysis of the data and in the writing of this paper. All authors read and approved the final manuscript.

\section{Competing interests}

ICNARC conducts paid for analyses for industry, including Eli Lilly and Company (Indianapolis, IN, USA). DAH and KMR are employees of ICNARC. $\mathrm{BHC}$ has received honoraria for consultancies and speaking from Eli Lilly and Company.

Received: 28 June 2011 Revised: 16 August 2011

Accepted: 23 September 2011 Published: 26 September 2011

\section{References}

1. Angus DC, Linde-Zwirble WT, Lidicker J, Clermont G, Carcillo J, Pinsky MR: Epidemiology of severe sepsis in the United States: analysis of incidence, outcome, and associated costs of care. Crit Care Med 2001, 29:1303-1310.

2. Brun-Buisson C, Doyon F, Carlet J, Dellamonica P, Gouin F, Lepoutre A, Mercier JC, Offenstadt G, Regnier B: Incidence, risk factors, and outcome of severe sepsis and septic shock in adults. A multicenter prospective study in intensive care units. French ICU Group for Severe Sepsis. JAMA 1995, 274:968-974.

3. Padkin A, Goldfrad C, Brady AR, Young D, Black N, Rowan K: Epidemiology of severe sepsis occurring in the first $24 \mathrm{hrs}$ in intensive care units in England, Wales, and Northern Ireland. Crit Care Med 2003, 31:2332-2338.

4. Lindenauer PK, Rothberg MB, Nathanson BH, Pekow PS, Steingrub JS: Activated protein $C$ and hospital mortality in septic shock: a propensitymatched analysis. Crit Care Med 2010, 38:1101-1107.

5. Finfer S, Bellomo R, Lipman J, French C, Dobb G, Myburgh J: Adultpopulation incidence of severe sepsis in Australian and New Zealand intensive care units. Intensive Care Med 2004, 30:589-596.

6. Harrison DA, Welch CA, Eddleston JM: The epidemiology of severe sepsis in England, Wales and Northern Ireland, 1996 to 2004: secondary analysis of a high quality clinical database, the ICNARC Case Mix Programme Database. Crit Care 2006, 10:R42.

7. Karlsson S, Varpula M, Ruokonen E, Pettila V, Parviainen I, Ala-Kokko TI, Kolho E, Rintala EM: Incidence, treatment, and outcome of severe sepsis in ICU-treated adults in Finland: the Finnsepsis study. Intensive Care Med 2007, 33:435-443.

8. Linde-Zwirble WT, Angus DC: Severe sepsis epidemiology: sampling, selection, and society. Crit Care 2004, 8:222-226.

9. Martin GS, Mannino DM, Eaton S, Moss M: The epidemiology of sepsis in the United States from 1979 through 2000. N Engl J Med 2003, 348:1546-1554.

10. Hofhuis JG, Spronk PE, van Stel HF, Schrijvers AJ, Rommes JH, Bakker J: The impact of severe sepsis on health-related quality of life: a long-term follow-up study. Anesth Analg 2008, 107:1957-1964.

11. Heyland DK, Hopman W, Coo H, Tranmer J, McColl MA: Long-term healthrelated quality of life in survivors of sepsis. Short Form 36: a valid and reliable measure of health-related quality of life. Crit Care Med 2000, 28:3599-3605.

12. Abraham E, Laterre PF, Garg R, Levy H, Talwar D, Trzaskoma BL, Francois B, Guy JS, Bruckmann M, Rea-Neto A, Rossaint R, Perrotin D, Sablotzki A, Arkins N, Utterback BG, Macias WL: Drotrecogin alfa (activated) for adults with severe sepsis and a low risk of death. N Engl J Med 2005, 353:1332-1341.

13. Bernard GR, Vincent JL, Laterre PF, LaRosa SP, Dhainaut JF, LopezRodriguez A, Steingrub JS, Garber GE, Helterbrand JD, Ely EW, Fisher CJ Jr, Recombinant human protein C Worldwide Evaluation in Severe Sepsis (PROWESS): Efficacy and safety of recombinant human activated protein C for severe sepsis. N Engl J Med 2001, 344:699-709.

14. US Food and Drug Administration: FDA clinical review, drotrecogin alfa (activated), [recombinant human activated protein C (rhAPC)], Xigris ${ }^{\mathrm{TM}}$. Washington, DC: US Food and Drug Administration; 2002 [http://www.fda. gov/downloads/drugs/developmentapprovalprocess/ howdrugsaredevelopedandapproved/approvalapplications/ therapeuticbiologicapplications/ucm193970.pdf].

15. Anti-Infective Advisory Committee, US Food and Drug Administration: FDA briefing document: Anti-Infective Advisory Committee, drotrecogin alfa 
(activated), [recombinant human activated protein C (rhAPC)], Xigris ${ }^{\mathrm{TM}}$. Washington, DC: US Food and Drug Administration; 2001 [http://www.fda. gov/ohrms/dockets/ac/01/briefing/3797b1_02_FDAbriefing.pdf].

16. Nadel S, Goldstein B, Williams MD, Dalton $H$, Peters M, Macias WL, AbdAllah SA, Levy H, Angle R, Wang D, Sundin DP, Giroir B, REsearching severe Sepsis and Organ dysfunction in children: a gLobal perspective (RESOLVE) study: Drotrecogin alfa (activated) in children with severe sepsis: a multicentre phase III randomised controlled trial. Lancet 2007, 369:836-843.

17. Finfer S, Ranieri VM, Thompson BT, Barie PS, Dhainaut JF, Douglas IS, Gardlund B, Marshall JC, Rhodes A: Design, conduct, analysis and reporting of a multi-national placebo-controlled trial of activated protein C for persistent septic shock. Intensive Care Med 2008, 34:1935-1947.

18. Angus DC, Linde-Zwirble WT, Clermont G, Ball DE, Basson BR, Ely EW, Laterre PF, Vincent JL, Bernard G, van Hout B: Cost-effectiveness of drotrecogin alfa (activated) in the treatment of severe sepsis. Crit Care Med 2003, 31:1-11.

19. Davies A, Ridley S, Hutton J, Chinn C, Barber B, Angus DC: Cost effectiveness of drotrecogin alfa (activated) for the treatment of severe sepsis in the United Kingdom. Anaesthesia 2005, 60:155-162.

20. Fowler RA, Hill-Popper M, Stasinos J, Petrou C, Sanders GD, Garber AM: Cost-effectiveness of recombinant human activated protein $C$ and the influence of severity of illness in the treatment of patients with severe sepsis. J Crit Care 2003, 18:181-191, discussion 191-184.

21. Green C, Dinnes J, Takeda AL, Cuthbertson BH: Evaluation of the costeffectiveness of drotrecogin alfa (activated) for the treatment of severe sepsis in the United Kingdom. Int J Technol Assess Health Care 2006, 22:90-100.

22. Manns BJ, Lee H, Doig CJ, Johnson D, Donaldson C: An economic evaluation of activated protein $C$ treatment for severe sepsis. N Engl J Med 2002, 347:993-1000.

23. Neilson AR, Burchardi H, Chinn C, Clouth J, Schneider H, Angus D: Costeffectiveness of drotrecogin alfa (activated) for the treatment of severe sepsis in Germany. J Crit Care 2003, 18:217-227.

24. Hodder RV, Hall R, Russell JA, Fisher HN, Lee B: Early drotrecogin alpha (activated) administration in severe sepsis is associated with lower mortality: a retrospective analysis of the Canadian ENHANCE cohort. Crit Care 2009, 13:R78.

25. Vincent JL, O'Brien J Jr, Wheeler A, Wittebole X, Garg R, Trzaskoma BL, Sundin DP: Use of an integrated clinical trial database to evaluate the effect of timing of drotrecogin alfa (activated) treatment in severe sepsis. Crit Care 2006, 10:R74.

26. Revicki DA, Frank L: Pharmacoeconomic evaluation in the real world. Effectiveness versus efficacy studies. Pharmacoeconomics 1999, 15:423-434.

27. Sculpher MJ, Claxton K, Drummond M, McCabe C: Whither trial-based economic evaluation for health care decision making? Health Econ 2006 15:677-687.

28. Sekhon J, Grieve R: A matching method for improving covariate balance in cost-effectiveness analysis. Health Economics 2011.

29. Rowan KM, Welch CA, North E, Harrison DA: Drotrecogin alfa (activated): real-life use and outcomes for the UK. Crit Care 2008, 12:R58.

30. Sadaka F, O'Brien J, Migneron M, Stortz J, Vanston A, Taylor RW: Activated protein C in septic shock: a propensity-matched analysis. Crit Care 2011, 15:R89.

31. Dhainaut JF: International integrated database for the evaluation of severe sepsis (INDEPTH): clinical evaluation committee report on the safety of drotrecogin alfa (activated) therapy. Curr Med Res Opin 2008, 24:1187-1197.

32. Laterre PF, Nelson DR, Macias W, Abraham E, Sashegyi A, Williams MD, Levy M, Levi M, Utterback B, Vincent JL: International integrated database for the evaluation of severe sepsis and drotrecogin alfa (activated) therapy: 28-day survival and safety. J Crit Care 2007, 22:142-152.

33. Martin G, Brunkhorst FM, Janes JM, Reinhart K, Sundin DP, Garnett K, Beale R: The international PROGRESS registry of patients with severe sepsis: drotrecogin alfa (activated) use and patient outcomes. Crit Care 2009, 13:R103.

34. Payen D, Sablotzki A, Barie PS, Ramsay G, Lowry S, Williams M, Sarwat S, Northrup J, Toland P, Booth FV: International integrated database for the evaluation of severe sepsis and drotrecogin alfa (activated) therapy: analysis of efficacy and safety data in a large surgical cohort. Surgery 2007, 141:548-561.

35. Sashegyi A, Trzaskoma BL, Nelson DR, Williams MD, Macias W: International INtegrated Database for the Evaluation of severe sePsis and drotrecogin alfa (activated) THerapy: component trials and statistical methods for INDEPTH. Curr Med Res Opin 2006, 22:1001-1012.

36. Ferrer R, Artigas A, Suarez D, Palencia E, Levy MM, Arenzana A, Perez XL, Sirvent JM: Effectiveness of treatments for severe sepsis: a prospective, multicenter, observational study. Am J Respir Crit Care Med 2009, 180:861-866

37. National Institute for Health and Clinical Excellence: Guide to the methods of technology appraisal. London: National Institute for Health and Clinical Excellence; 2008 [http://www.nice.org.uk/media/B52/A7/

TAMethodsGuideUpdatedJune2008.pdf].

38. Harrison DA, Brady AR, Rowan K: Case mix, outcome and length of stay for admissions to adult, general critical care units in England, Wales and Northern Ireland: the Intensive Care National Audit \& Research Centre Case Mix Programme Database. Crit Care 2004, 8:R99-111.

39. DocDat: The directory of clinical databases [http://www.icapp.nhs.uk/docdat/ ].

40. Wright JC, Plenderleith L, Ridley SA: Long-term survival following intensive care: subgroup analysis and comparison with the general population. Anaesthesia 2003, 58:637-642.

41. National Institute for Health and Clinical Excellence: Drotrecogin alpha (activated) for severe sepsis. London: National Institute for Health and Clinical Excellence; 2004 [http://guidance.nice.org.uk/TA84].

42. Department of Health: NHS reference costs 2009-2010. London: Department of Health; 2011.

43. Curtis L: Unit costs of health \& social care 2010. Canterbury, UK: Personal Social Services Research Unit, University of Kent; 2010 [http://www.pssru.ac uk/pdf/uc/uc2010/uc2010.pdf].

44. Leventhal H, Colman S: Quality of life: a process view. Psychol Health 1997, 12:753-767.

45. Cuthbertson BH, Roughton S, Jenkinson D, Maclennan G, Vale L: Quality of life in the five years after intensive care: a cohort study. Crit Care 2010, 14:R6.

46. Soares MO, Welton NJ, Harrison DA, Peura P, Shankar HM, Harvey SE, Madan JJ, Ades AE, Palmer SJ, Rowan KM: An evaluation of the feasibility, cost and value of information of a multicentre randomised controlled trial of intravenous immunoglobulin for sepsis (severe sepsis and sepsis shock): incorporating a systematic review, meta analysis and value of information analysis. Health Technology Assessment 2011.

47. Green C, Dinnes J, Takeda A, Shepherd J, Hartwell D, Cave C, Payne E, Cuthbertson BH: Clinical effectiveness and cost-effectiveness of drotrecogin alfa (activated) (Xigris) for the treatment of severe sepsis in adults: a systematic review and economic evaluation. Health Technol Assess 2005, 9:1-126, iii-iv.

48. Cuthbertson BH, Scott J, Strachan M, Kilonzo M, Vale L: Quality of life before and after intensive care. Anaesthesia 2005, 60:332-339.

49. Dolan P, Gudex C, Kind P, Williams A: A social tariff for EuroQol: results from a UK general population survey. Centre for Health Economics Discussion Paper 1995, 138:1-19.

50. Young JD, Goldfrad C, Rowan K: Development and testing of a hierarchical method to code the reason for admission to intensive care units: the ICNARC Coding Method. Intensive Care National Audit \& Research Centre. Br J Anaesth 2001, 87:543-548.

51. Buis ML: Using and interpreting restricted cubic splines. Tübingen, Germany: Institut für Soziologie, Eberhard Karls Universität Tübingen; 2009 [http://fmwww.bc.edu/repec/dsug2009/buis.pdf].

52. Grieve R, Sekhon JS, Hu TW, Bloom JR: Evaluating health care programs by combining cost with quality of life measures: a case study comparing capitation and fee for service. Health Serv Res 2008, 43:1204-1222.

53. Gilligan MJ, Sargenti EJ: Do UN interventions cause peace? Using matching to improve causal interference. Quarterly Journal of Political Science 2008, 3:89-122.

54. Gordon SC, Huber GA: The effect of elecoral competitiveness on incumbent behaviour. Quarterly Journal of Political Science 2007, 2:107-138.

55. Heron MC, Wand J: Assessing partisan bias in voting technology: the case of the 2004 New Hamshire recount. Elect Stud 2007, 26:247-261.

56. Imbens GM, Wooldridge JM: Recent developments in the econometrics of program evaluation. Journal of Economic Literature 2009, 47:5-86. 
57. Austin PC: Balance diagnostics for comparing the distribution of baseline covariates between treatment groups in propensity-score matched samples. Stat Med 2009, 28:3083-3107.

58. Normand ST, Landrum MB, Guadagnoli E, Ayanian JZ, Ryan TJ, Cleary PD, McNeil BJ: Validating recommendations for coronary angiography following acute myocardial infarction in the elderly: a matched analysis using propensity scores. J Clin Epidemiol 2001, 54:387-398.

59. Stinnett AA, Mullahy J: Net health benefits: a new framework for the analysis of uncertainty in cost-effectiveness analysis. Med Decis Making 1998, 18:568-80.

60. Davison AC, Hinkley DV: Bootstrap Methods and Their Application. New York: Cambridge University Press; 1997.

61. Fenwick E, O'Brien BJ, Briggs A: Cost-effectiveness acceptability curvesfacts, fallacies and frequently asked questions. Health Econ 2004, 13:405-415.

62. Eli Lilly and Company: Xigris NICE submission: drotrecogin alfa (activated) for severe sepsis. Basingstoke, UK: Eli Lilly and Company; 2003.

63. Camporota L, Wyncoll D: Practical aspects of treatment with drotrecogin alfa (activated). Crit Care 2007, 11(Suppl 5):S7

64. Dhainaut JF, Payet $S$, Vallet $B$, Franca $L R$, Annane $D$, Bollaert PE, Le Tulzo $Y$, Runge I, Malledant Y, Guidet B, Le Lay K, Launois R, PREMISS Study Group: Cost-effectiveness of activated protein $C$ in real-life clinical practice. Crit Care 2007, 11:R99.

doi:10.1186/cc10468

Cite this article as: Sadique et al:: Is Drotrecogin alfa (activated) for adults with severe sepsis, cost-effective in routine clinical practice? Critical Care 2011 15:R228.

\section{Submit your next manuscript to BioMed Central} and take full advantage of:

- Convenient online submission

- Thorough peer review

- No space constraints or color figure charges

- Immediate publication on acceptance

- Inclusion in PubMed, CAS, Scopus and Google Scholar

- Research which is freely available for redistribution

Submit your manuscript at www.biomedcentral.com/submit
Biomed Central 